\title{
Desempeño de turbinas eólicas Magnus de eje horizontal en función de sus variables geométricas y cinemáticas
}

Horizontal axis Magnus wind turbine performance according to their geometric and kinematic variables

Gustavo Richmond-Navarro ${ }^{1}$

Fecha de recepción: 29 de mayo del 2015

Fecha de aprobación: 7 de setiembre del 2015

Richmond-Navarro, G. Desempeño de turbinas eólicas Magnus de eje horizontal en función de sus variables geométricas y cinemáticas. Tecnología en Marcha. Vol. 29, № 1, Enero-Marzo.

Pág 38-50. 


\section{Palabras clave}

Turbina eólica Magnus; efecto Magnus; desempeño; método numérico.

\section{Resumen}

Este estudio presenta el análisis de una turbina eólica de eje horizontal que utiliza cilindros en rotación, en lugar de aspas con perfiles alares. El principio de funcionamiento de este generador eólico es el efecto Magnus, que sucede cuando las aspas cilíndricas empiezan a rotar y se da una interacción entre la corriente de viento incidente y el aire que es arrastrado por las paredes de los cilindros en movimiento. De esta forma, se obtiene la sustentación que pone en movimiento la turbina.

El objetivo de la investigación es caracterizar este tipo de turbina mediante modelos numéricos y matemáticos, que permitan determinar el comportamiento de la curva de potencia en función del viento ante variaciones de los parámetros de funcionamiento de la turbina tipo Magnus de eje horizontal, a saber, la geometría del cilindro y las velocidades de rotación tanto de la turbina como del cilindro.

Para estudiar el desempeño de la turbina, se propone un método numérico no iterativo, que es implementado en un código que permite predecir el rendimiento de turbinas de eje horizontal, el cual es validado con mediciones experimentales de turbinas convencionales.

Posteriormente, se adecúa el código para aplicarlo a turbinas Magnus y con ello se obtiene el comportamiento de la curva de potencia ante variaciones en la geometría y cantidad de cilindros, así como las velocidades angulares de la turbina y del aspa cilíndrica.

\section{Keywords}

Magnus wind turbine; Magnus effect; performance; numerical method.

\section{Abstract}

This study covers the analysis of a horizontal axis wind turbine that uses rotating cylinders instead of blades. The working principle of this wind generator is the Magnus effect, which happens when the cylinders start rotating, giving rise to an interaction between the incident wind and the air dragged by the walls of the moving cylinders. This generates lift which puts the turbine in motion.

The goal of this investigation was to characterize this type of turbine by means of numerical and mathematics methods that permit determination of the power vs wind speed curve's behaviour as a function of working parameters of Magnus horizontal axis wind turbines.

In order to study turbines performance, a non-iterative method is proposed and implemented in code. This approach allows the prediction of the output power, which is validated by experimental measurements of conventional turbines.

The method was adapted to Magnus turbines. It was used to obtain the power curve behavior given geometry variations including changes in the number of rods, as well as turbine and cylindrical blade's angular velocities. 


\section{Introducción}

En la actualidad, la creciente demanda de energía ha impulsado diversas e innovadoras maneras de aprovechar el recurso eólico para la producción de electricidad. Fei, Mai y Li (2012) describen un sistema de recolección de energía eólica basado en las vibraciones mecánicas que se inducen en una cinta flexible debido a la interacción del fluido con la estructura. Perkovic, Silva, Ban, Kranjčević y Duić (2013) presentan un mecanismo que consiste en un volantín diseñado para transformar la energía cinética del viento, a gran altitud, en energía mecánica.

Por otra parte, las turbinas eólicas convencionales (artefactos que extraen energía cinética del viento) están en constante rediseño e investigación para obtener cada vez mejores productos en cuanto a eficiencia, confiabilidad y armonía con el entorno (Burton, Sharpe, Jenkins \& Bossanyi, 2011).

Las turbinas eólicas de eje horizontal son las más comúnmente diseñadas hoy en día (Manwel, McGowan \& Rogers, 2009). Poseen una torre que soporta el eje de rotación a una determinada altura sobre el suelo y paralelo a este, de modo que se aprovecha la energía del viento que viaja a una velocidad mayor en tanto más alta sea la torre que soporta a la turbina.

Existe una amplia gama de formas de turbinas de eje vertical; entre ellas destaca la Darrieus, que ampliamente fue estudiada en Estados Unidos y Canadá en las décadas de 1970 y 1980 (Manwel et al., 2009). Aún hoy en día se presentan modelos matemáticos y computacionales para el análisis de este tipo de turbinas (Islam, Ting \& Fartaj, 2008).

En el campo de las turbinas eólicas de eje horizontal, recientemente se ha explorado la alternativa de utilizar, en lugar de aspas, cilindros girando que aprovechan el efecto Magnus para generar la fuerza de sustentación (Bychkov, Dovgal \& Kozlov, 2007). La figura 1 muestra el aerogenerador conocido como turbinas Magnus, mostrando sus parámetros geométricos y cinemáticos (diámetro $D$ y longitud $L$ del cilindro, velocidad angular de la turbina $\Omega$ y del cilindro $\omega$ ) así como el viento incidente $U_{\infty}$.

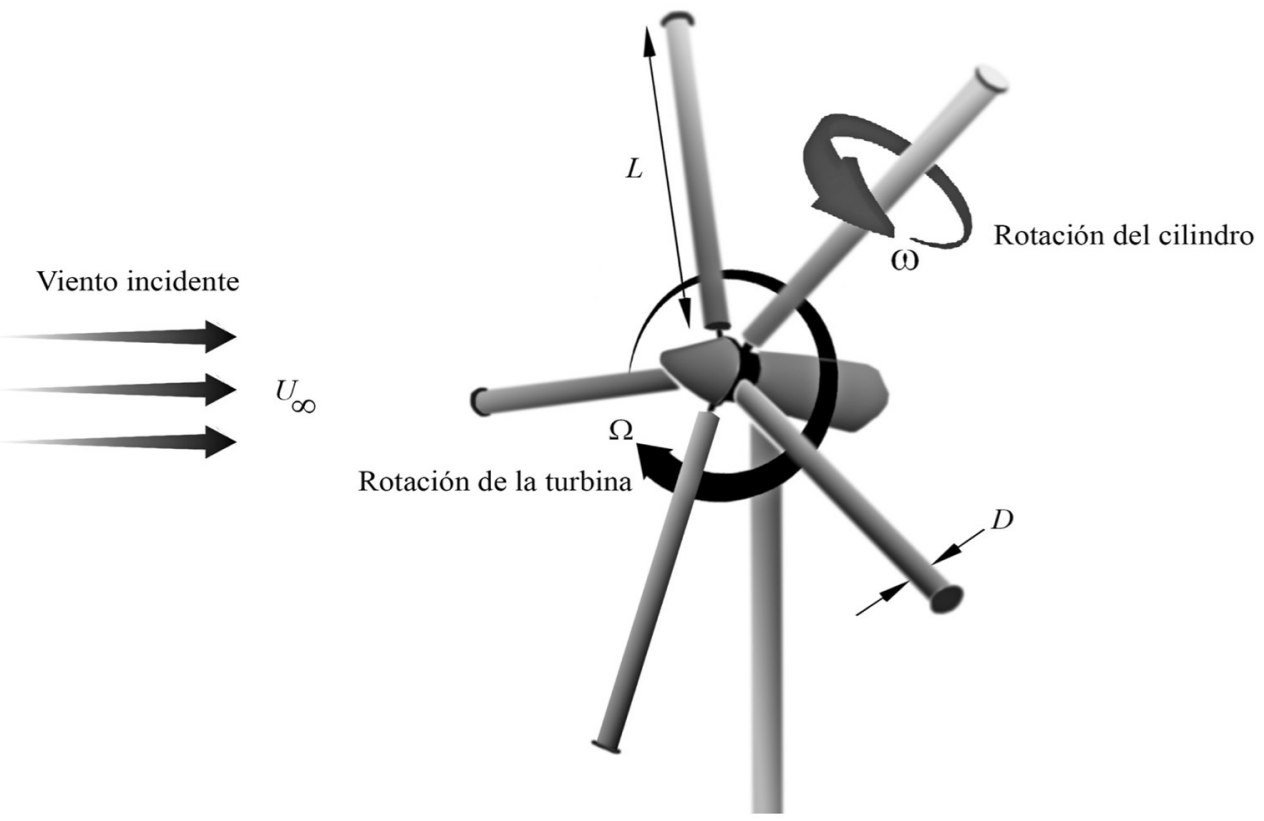

Figura 1. Turbina eólica Magnus de eje horizontal. 
El efecto Magnus es una fuerza que actúa sobre un cuerpo en rotación sumergido en un fluido en movimiento; la dirección de esta fuerza es perpendicular a la dirección del flujo, según se muestra en la figura 2. Esto se debe a una diferencia de presión causada por la diferencia de velocidad que existe en el fluido entre la cara superior e inferior; la modificación en el campo de velocidades sucede al girar el cilindro arrastrando consigo el fluido a su alrededor por la condición de no deslizamiento.

Bychkov et al. (2007) presentan una investigación teórica y experimental que propone la operación óptima de una turbina eólica que utiliza cilindros girando en lugar de aspas. Sus resultados para un cilindro en rotación muestran un crecimiento lineal para el coeficiente de sustentación $C_{L}$, que es muy superior al correspondiente de un perfil alar.

Ito, Kawashima, Nishizawa, Ushiyama \& Komatinovic (2007) fabrican un prototipo de una turbina Magnus-Savonius, en la que los cilindros de la turbina Magnus son reemplazados por rotores Savonius. De este modo, se evita la necesidad de hacer girar los cilindros con energía externa, pues los rotores Savonius generarían su propia rotación por arrastre. Una de sus conclusiones es que este aparato tendrá un coeficiente de potencia mucho menor al de turbinas similares con aspas, pero a cambio el costo de fabricación es muy bajo.

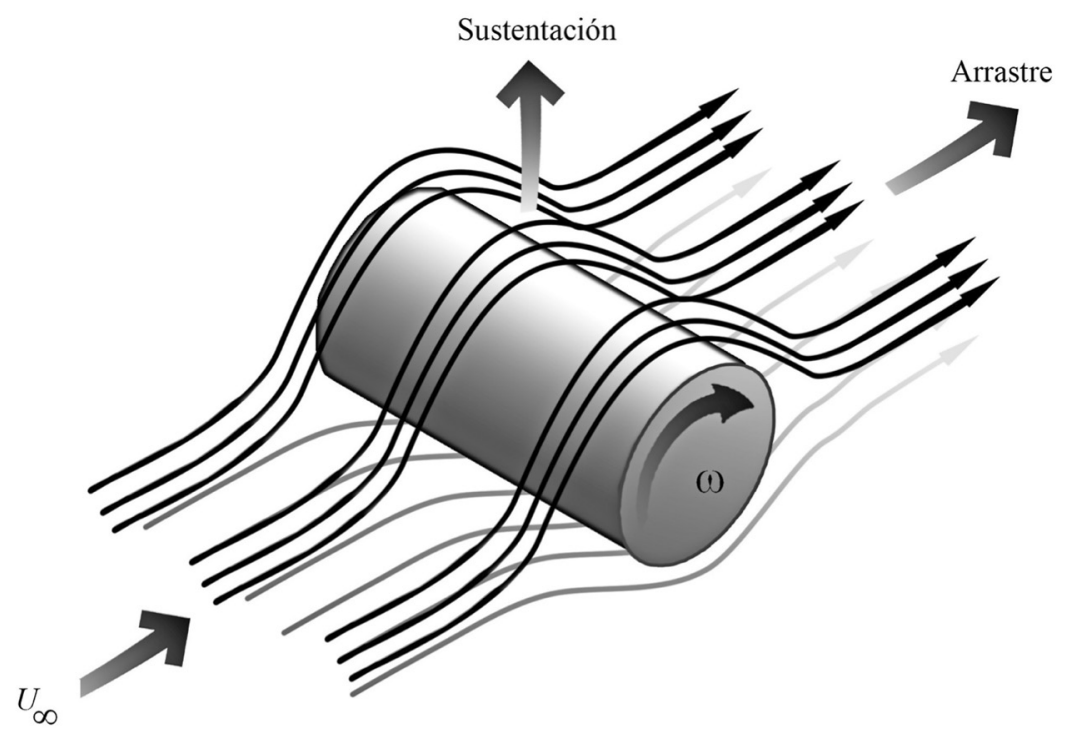

Figura 2. Efecto Magnus.

Bychkov (2008) presenta una alternativa para la turbina Magnus, en la cual la base del cilindro no gira. En este caso, emplea tapas laterales en los cilindros, para suprimir la vorticidad en las puntas de estos.

Gono, Rusek \& Hrabčík (2009) describen la turbina Magnus manufacturada por la empresa Mecaro Co. Destacan que en ese modelo se emplean cilindros con aletas espirales, para potenciar el efecto Magnus generado con la rotación de los cilindros.

Luo, Huang \& Wu (2011) presentan una solución analítica para el desempeño de una turbina Magnus. Desprecian el efecto del arrastre para el cálculo del torque desarrollado por los cilindros, por lo que su modelo corresponde a una turbina en un flujo no viscoso. 
Sun, Zhuang, Cao, Huang \& Wu (2012) Ilevan a cabo una simulación numérica para estudiar el efecto de modificar la forma de los cilindros en una turbina Magnus, empleando ANSYS CFX. La simulación se lleva a cabo con solo un cilindro, aprovechando la periodicidad rotacional del problema e imponiendo adecuadamente las condiciones de borde. Utilizan el modelo de turbulencia SST $k$ - $\omega$ por su precisión en el tratamiento de la capa límite ante gradientes de presión adversos. Obtienen que un cono truncado, con radio creciente alejándose del eje de rotación de la turbina, produce un coeficiente de potencia mayor que un cilindro circular. Pero para esto requiere mayores valores de $\omega$.

Sedaghat (2014) reporta que para el caso de la turbina Magnus resulta clave lograr la reducción de la relación entre arrastre y sustentación, si se busca mejorar la eficiencia.

Massaguer, Massaguer, Pujol, Comamala y Velayos (2014) proponen generar la rotación de los cilindros de la turbina Magnus por efectos centrífugos y autorotación, evitando la necesidad de invertir energía externa para generar el efecto Magnus. Entre otros aspectos, sus pruebas concluyen que este tipo de turbina genera menos ruido que las convencionales.

La revisión bibliográfica evidencia que la turbina Magnus ha sido objeto de estudio durante los últimos años. Uno de los resultados es que la turbina tiene eventuales ventajas respecto de las tradicionales que usan aspas. No obstante, ningún autor presenta una curva de potencia, teórica o experimental, para una turbina Magnus, ni su comportamiento ante modificaciones geométricas o cinemáticas; estos aspectos forman parte del presente estudio y en eso radica su originalidad e innovación.

\section{Metodología}

\section{Modelamiento y método propuesto}

La teoría del Disco Actuador, descrito en detalle por Burton et al. (2011), explica que el viento disminuye su velocidad al atravesar un generador eólico. Para modelar el intercambio de energía cinética entre el aire y una turbina eólica se define el factor de inducción axial $a$, el cual es una medida de qué tanto disminuyó su velocidad el viento al salir de la turbina y, por ende, cuantifica cuánta energía entregó el aire al generador.

Mediante argumentos de conservación de momentum lineal y la ecuación de Bernoulli, Burton et al. (2011) demuestran que la fuerza horizontal (denotada como $T$, del inglés Thrust) ejercida por la turbina al aire y viceversa es:

$$
T=2 \rho U_{\infty}^{2}(a(1-a)) A_{d}
$$

Donde $U_{\infty}$ representa la velocidad del viento libre, lejos de la turbina, $\rho$ es la densidad del fluido y $A_{d}$ es el área del disco que barren las aspas.

Este disco puede ser analizado en pequeños anillos, tal como lo propone la teoría BEM (Blade Element Momentum) descrita en detalle por Burton et al. (2011). Al realizar este tipo de análisis, la ecuación 1 puede ser escrita para un anillo diferencial a una distancia r como:

$$
d T=2 \rho U_{\infty}^{2}(a(1-a)) \delta A_{d}=4 \rho U_{\infty}^{2}(a(1-a)) \pi r \delta r
$$

La ecuación 2 es válida para $a<0.4$ y dentro de ella se tiene el coeficiente de empuje $\left(C_{T}\right)$.

$$
C_{T}=4 a(1-a) \quad \text { con } \quad a<0.4
$$


Por consideraciones de conservación de momentum angular, Burton et al. (2011) obtienen que el diferencial de torque $Q$ generado por el viento en cada disco es:

$$
\delta Q=\rho U_{\infty}(1-a) 2 \Omega a^{\prime} r^{2} \delta A_{d}=4 \rho U_{\infty} a^{\prime}(1-a) \Omega \pi r^{3} d r
$$

Donde $a^{\prime}$ es el factor de inducción tangencial, que modela el cambio en la velocidad tangencial del viento al atravesar la turbina eólica. Aguas arriba el viento viaja horizontalmente, pero dado que interacciona con la turbina y la hace girar, se genera una fuerza de reacción que dota al viento de velocidad tangencial aguas abajo de la turbina.

La ecuación 2 pierde validez cuando la turbina opera en un estado de estela turbulenta, que ocurre cuando $a>0.4$. Para compensar este efecto, se emplean dos correcciones anidadas (Moriarty y Hansen, 2005). La de Glauert reemplaza el $C_{T}=4 a(1-a)$ por:

$$
C_{T}=\frac{8}{9}+\left(4 F-\frac{40}{9}\right) a+\left(\frac{50}{9}-4 F\right) a^{2} \quad \text { con } \quad a>0.4
$$

Donde Festá definido según el modelo de corrección de puntas de la siguiente manera:

$$
F=\frac{2}{\pi} \cos ^{-1}\left(e^{-f}\right) \text { con } f=\frac{B}{2} \frac{L-r}{r \operatorname{sen}(\alpha)}
$$

Aquí $B$ representa la cantidad de aspas que componen la turbina y, de acuerdo con la figura 3, $\alpha$ se escribe como:

$$
\alpha=\operatorname{atan}\left(U_{\infty}(1-a) / \Omega r\left(1+a^{\prime}\right)\right)
$$

Esta corrección de puntas también se debe aplicar de forma directa en las ecuaciones 2 y 4 , lo que resulta de la siguiente forma:

$$
\begin{gathered}
\delta T=C_{T} \rho U_{\infty}^{2} \pi F r \delta r \\
\delta Q=4 \rho U_{\infty} a^{\prime}(1-a) \Omega \pi F r^{3} \delta r
\end{gathered}
$$

Por otra parte, las velocidades y fuerzas aerodinámicas que actúan sobre un elemento de aspa tal como se muestra en la figura 3, permiten escribir expresiones para $T$ y $Q$ en función de las magnitudes de las fuerzas de sustentación $F_{L}$ y arrastre $F_{D^{*}}$. No obstante, estas dos fuerzas pueden ser escritas en función de los coeficientes adimensionales $C_{L}$ y $C_{D}$ de forma diferencial (Burton et al., 2011) como:

$$
\delta F_{L}=\frac{1}{2} \rho U_{n}^{2} c C_{L} \delta r \quad ; \quad \delta F_{D}=\frac{1}{2} \rho U_{n}^{2} c C_{D} \delta r
$$

Donde $c$ es la cuerda del aspa y $U_{n}$ se obtiene de la figura 3. 


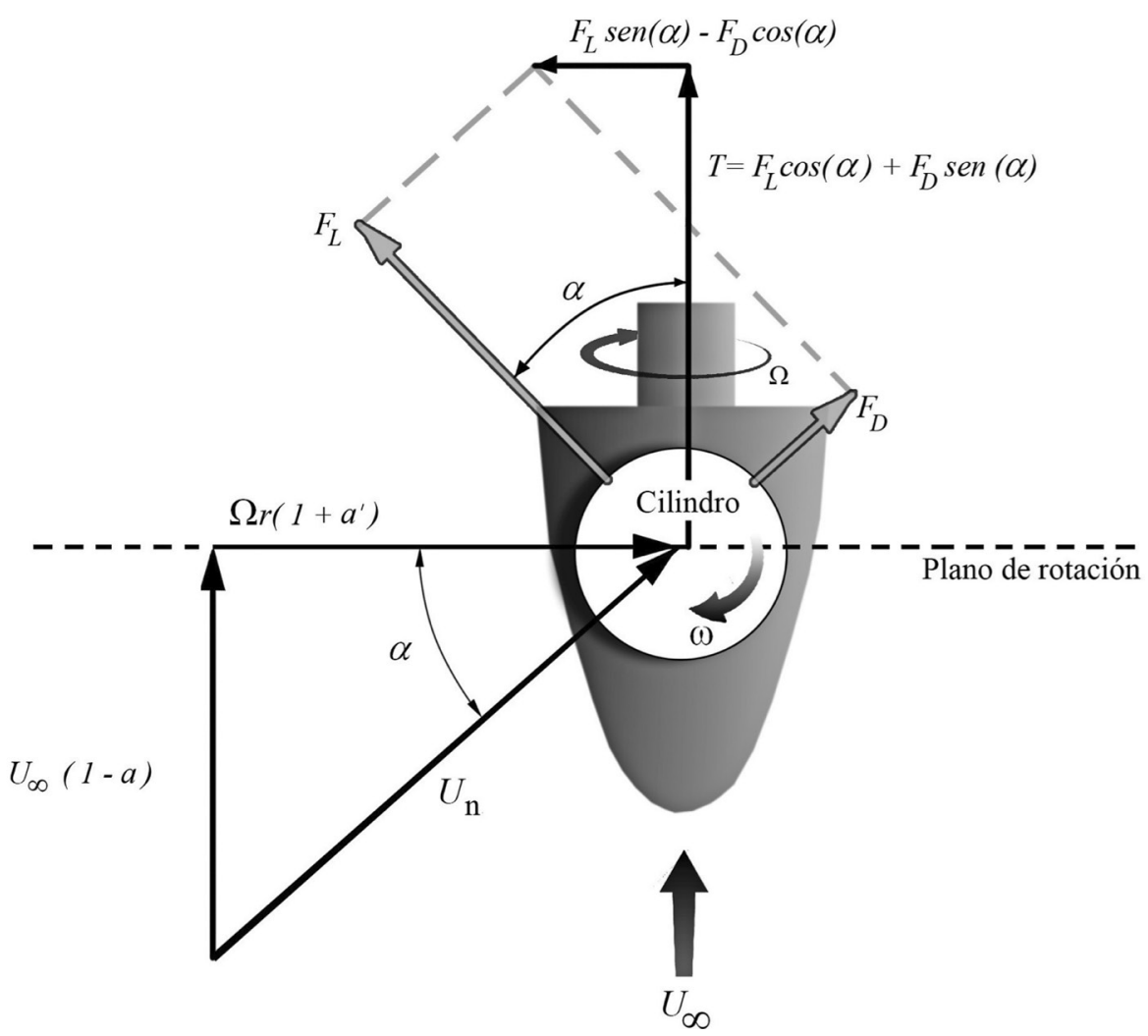

Figura 3. Acción del viento y fuerzas aerodinámicas sobre un aspa cilíndrica.

Para determinar los factores de inducción axial y tangencial se combinan las expresiones para la fuerza horizontal según la conservación de momentum lineal (ecuación 8), el torque según la conservación de momentum angular (ecuación 9) y las fuerzas aerodinámicas (figura 3 y ecuación 10) para obtener:

$$
\begin{gathered}
C_{T} \rho U_{\infty}^{2} \pi F r \delta r=\frac{1}{2} \rho U_{n}^{2}\left(C_{L} \cos (\alpha)+C_{D} \operatorname{sen}(\alpha)\right) B c \delta r \\
4 a^{\prime}(1-a) \rho U_{\infty} \Omega r^{3} \pi F \delta r=\frac{1}{2} \rho U_{n}^{2}\left(C_{L} \operatorname{sen}(\alpha)-C_{D} \cos (\alpha)\right) B r c \delta r
\end{gathered}
$$

Las ecuaciones 11 y 12 se reescriben de manera que todos los términos estén a un lado del igual, del otro lado se colocan los residuos $R 1$ y $R 2$, que idealmente deben ser igual a cero. Pero al igualar dos modelos que toman en cuenta simplificaciones y desprecian efectos tridimensionales y turbulencia entre otros, se debe buscar el punto en el que ambos residuos alcancen un mínimo. De modo que se discretiza el disco actuador en anillos, y para cada posición $r$ se buscan los valores de $a$ y $a^{\prime}$, que se determinan cuando se cumple que:

$$
\min _{a, a^{\prime}}(|R 1|+|R 2|)
$$

El método que se popone e implementa en un código para resolver la ecuación 13 es no iterativo, por tanto se le ha llamado método directo y consiste en evaluar todas las posibles combinaciones de $a$ y $a^{\prime}$ en la ecuación 13, considerando que ambas pueden tomar valores entre 0 y 1 . El producto de esta evaluación es una superficie que representa el valor de la suma 
de los residuos, que están asociados a fuerza y momento que predice de forma no compensada alguno de los dos modelos empleados.

La solución válida para el sistema es el punto donde la superficie de residuo total alcanza el valor mínimo posible. Por lo que una vez evaluadas todas las combinaciones, un algoritmo extrae de forma directa los factores de inducción axial y tangencial que finalmente gobiernan el rendimiento de la turbina.

Luego de resolver la ecuación 13 para cada valor de $r$, se determina la potencia $P$ :

$$
\int_{0}^{L} d P=\int_{0}^{L} \Omega d Q=\int_{0}^{L} 4 a^{\prime}(1-a) \rho U_{\infty} \Omega^{2} r^{3} \pi d r
$$

Una vez discretizado el disco en fracciones de radio, se calcula la potencia como:

$$
P=\sum_{\Delta r}^{L} 4 a^{\prime}(1-a) \rho U_{\infty} \Omega^{2} r^{3} \pi \Delta r
$$

El proceso descrito se debe repetir para todo el rango de velocidad de viento en estudio.

\section{Validación del método propuesto}

Para validar el método directo propuesto se han utilizado como datos de entrada las características de la turbina Fase II (Ceyhan, 2010) del NREL (Laboratorio Nacional de Energía Renovable de EE.UU.), que se compone de tres aspas de $0.4572 \mathrm{~m}$ de cuerda, girando a 71.3 revoluciones por minuto (RPM), con un diámetro de $10.06 \mathrm{~m}$ y una potencia de $19.8 \mathrm{~kW}$. Los resultados comparativos de los valores predichos por el método directo y los valores reales de la turbina se muestran en la figura 4.

Se encuentra una adecuada concordancia entre los resultados obtenidos por el método directo propuesto y las mediciones experimentales para la turbina Fase II. Se aprecia una ligera sobreestimación de la potencia para velocidades de viento bajas y altas, menores a $9 \mathrm{~m} / \mathrm{s}$ y mayores a $19 \mathrm{~m} / \mathrm{s}$. En el rango restante, el método directo presenta una subestimación en la predicción de potencia. No obstante, se captura adecuadamente la tendencia de la curva de potencia y sus valores puntuales, considerando que el modelo utilizado no toma en cuenta efectos como rugosidad, pandeo y vibración de las aspas o turbulencia y variación del ángulo de incidencia lateral del viento, entre otros.

\section{Adecuación del método directo para turbinas Magnus}

El análisis de la turbina Magnus se lleva a cabo con el método directo validado anteriormente. Las dos diferencias fundamentales que implican una modificación en el planteamiento del modelo son la geometría, al reemplazar el perfil alar por un cilindro, y la rotación del cilindro.

Es por esto que en las ecuaciones 11 y 12, el concepto de cuerda debe ser sustituido por el diámetro del cilindro. Por otra parte, los coeficientes $C_{L}$ y $C_{D}$ son independientes del ángulo de ataque, dada la simetría cilíndrica de sus aspas; estos coeficientes, en el caso de la turbina Magnus, son función de la velocidad relativa de rotación del cilindro $\bar{\omega}=\omega r_{d} U_{\infty}$.

Para estudiar el desempeño de la turbina Magnus se emplea el método directo propuesto, prestando especial atención a la selección de las curvas de $C_{L}$ y $C_{D}$, pues son radicalmente distintas para un cilindro en comparación con cualquier perfil aerodinámico y, por ende, contienen la clave para lograr posibles mejoras respecto de las turbinas tradicionales en algún campo de aplicación particular. 


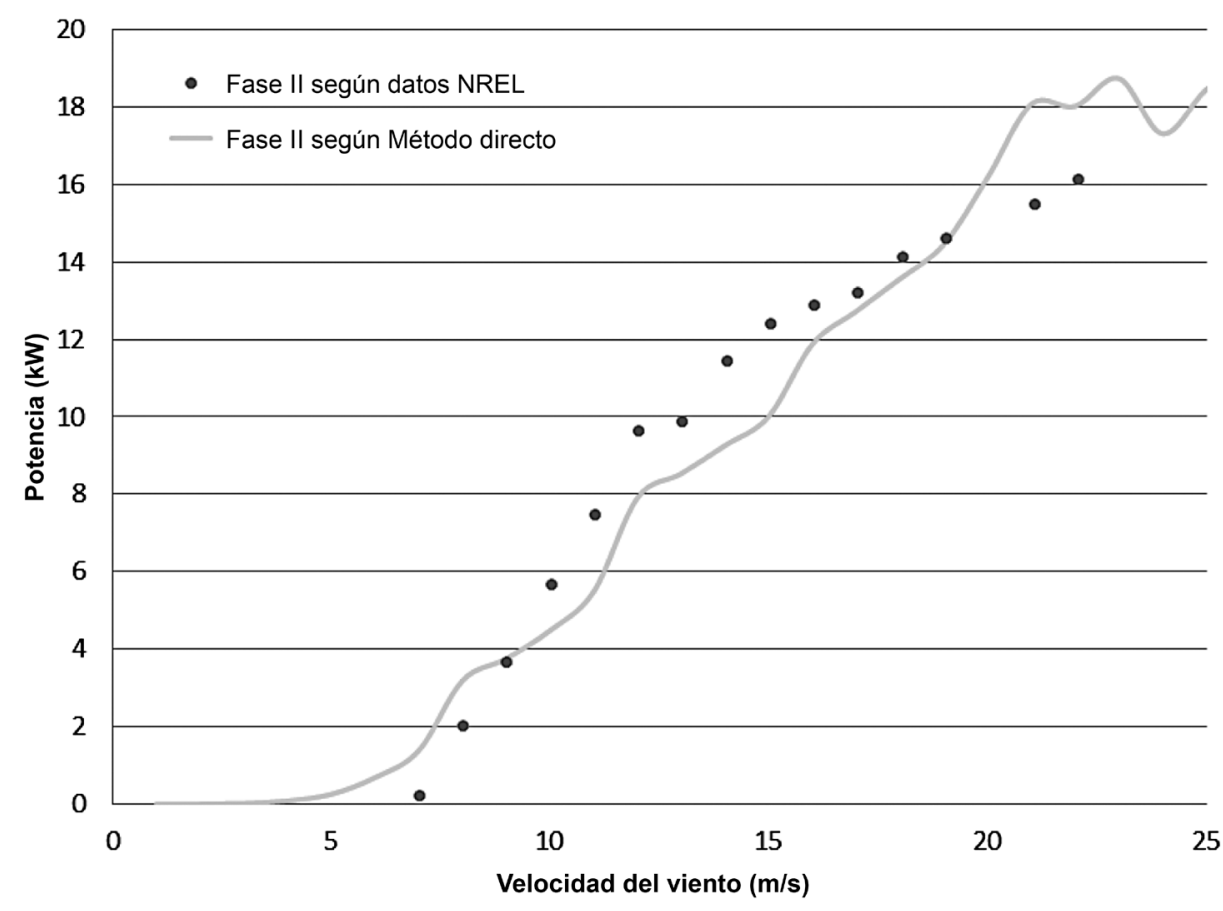

Figura 4. Potencia de la turbina NREL Fase II en función de la velocidad del viento.

\section{Resultados y discusión}

Se programa el método propuesto en el software comercial Matlab y se toma como dato de entrada las curvas de $C_{L}$ y $C_{D}$ según las mediciones experimentales de Bychkov et al. (2007).

Los resultados de las simulaciones realizadas mediante el método directo, para una turbina de 2 cilindros, se muestran en la figura 5, donde se han combinado cilindros de 15 y $25 \mathrm{~cm}$ de diámetro con velocidades de rotación en el rango de 10 a 4000 RPM y con turbinas de 1 a 3 m de radio con velocidades angulares de entre 10 y 30 RPM.

El principal hallazgo de esta combinación de variables es que, en general, se requiere alta velocidad de rotación del cilindro, del orden de $10^{3} \mathrm{RPM}$, para lograr extraer potencia del viento, dado que las líneas celeste y rosado ( $\omega=10$ y 100 RPM, respectivamente) están sobre el eje de cero potencia en todos los arreglos de variables para turbinas pequeñas.

Por esta razón, en la figura 6 se presenta el resultado de una nueva combinación de parámetros donde $\omega=1,4,8$ y $12 \times 10^{3}$ RPM, con el fin de determinar el comportamiento de la curva de potencia en relación con el cambio en todas las otras variables.

Con este nuevo escenario es posible definir la tendencia de la curva de potencia de la turbina Magnus. Se destaca que al aumentar la velocidad angular de los cilindros, el parámetro al que es más sensible el sistema, aumenta la potencia promedio y su punto máximo en relación con la velocidad del viento incidente.

La necesidad de una alta velocidad de rotación del cilindro se debe a que la curva del coeficiente de sustentación del cilindro en rotación no se inicia en el origen de coordenadas, sino que para valores menores a $\bar{\omega}=0.5$ se obtiene $C_{L}=0$ (Bychkov et al., 2007). 

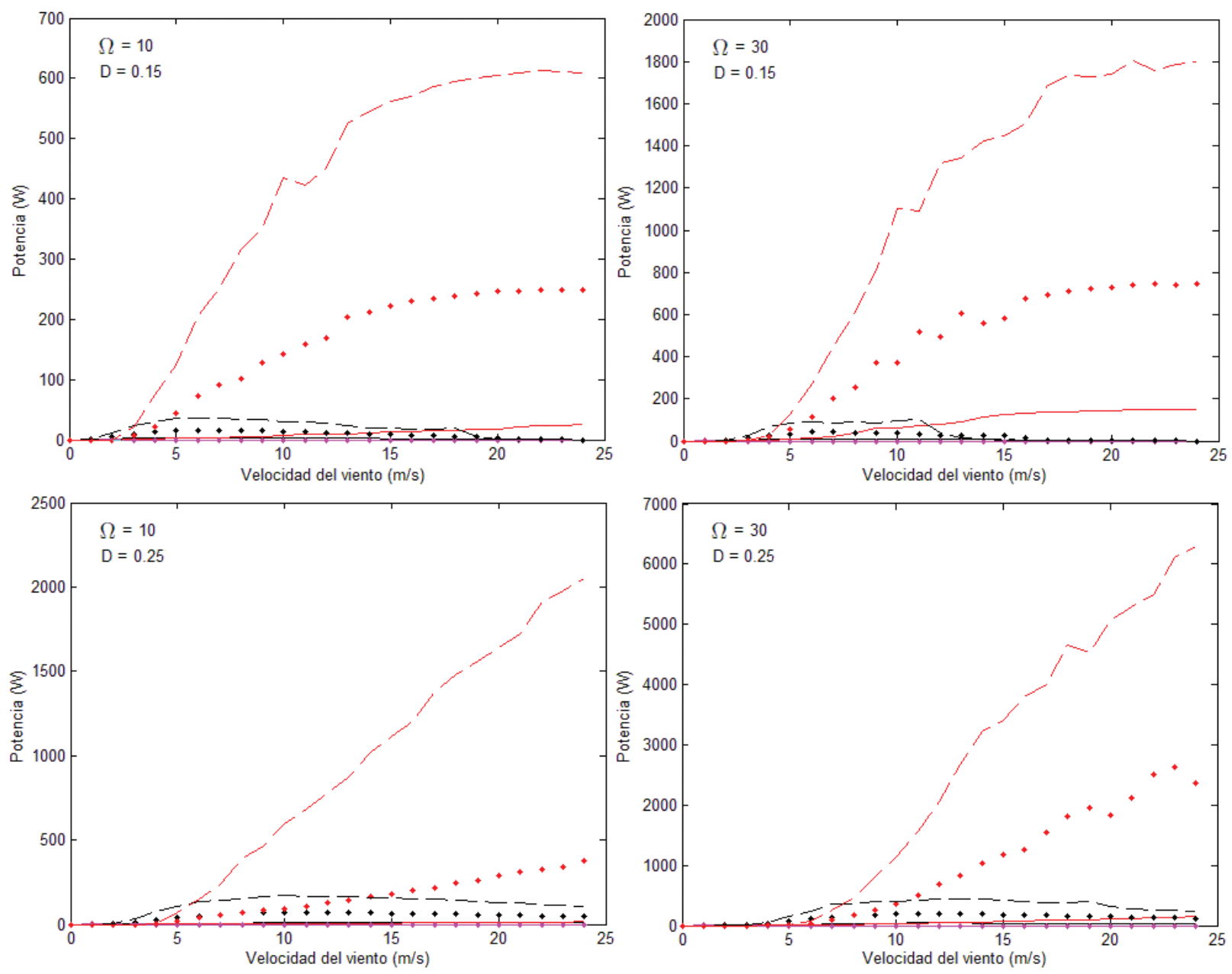

Figura 5. Curvas de potencia de la turbina Magnus de dos cilindros. En cada gráfico L = $1 \mathrm{~m}$ (sólida), $2 \mathrm{~m}$ (puntos) y 3 m (discontinua); $\omega=10$ RPM (celeste), 100 RPM (rosado), 1000 RPM (negro) y 4000 RPM (rojo). D (m), $\Omega$ (RPM)

Además, para que exista el efecto Magnus se requiere un adecuado balance entre la velocidad del viento incidente y la rotación del cilindro. Por una parte, una corriente de viento muy tenue no genera ninguna sustentación, por otro lado, tampoco habrá sustentación si la velocidad del viento es excesiva, en ese caso resulta despreciable la diferencia de velocidad del viento en las caras del cilindro y el sistema se puede aproximar como si el cuerpo cilíndrico no estuviese girando.

Por otra parte, si el cilindro gira a muy baja velocidad angular, no modifica las líneas de corriente del viento y, por ende, no se genera sustentación, pero también se obtiene cero sustentación si el cilindro gira con una velocidad angular excesiva. En este caso, el fenómeno se debe a que en la vecindad del cilindro las líneas de corriente corresponden prácticamente a las de un cilindro girando dentro de un fluido en reposo y no es posible percibir el efecto del flujo de viento externo.

Considerando que $\bar{\omega}$ combina la velocidad del viento con la velocidad angular del cilindro y que según estudios previos con $\bar{\omega}=2$ se obtiene la potencia máxima de la turbina, es razonable que al aumentar la velocidad angular de los cilindros se incremente la potencia capturada, pues se genera más sustentación y a la vez que el punto de máxima potencia ocurre para una velocidad mayor del viento incidente, ya que se debe mantener el balance para mantener constante el adimensional $\bar{\omega}=2$. 

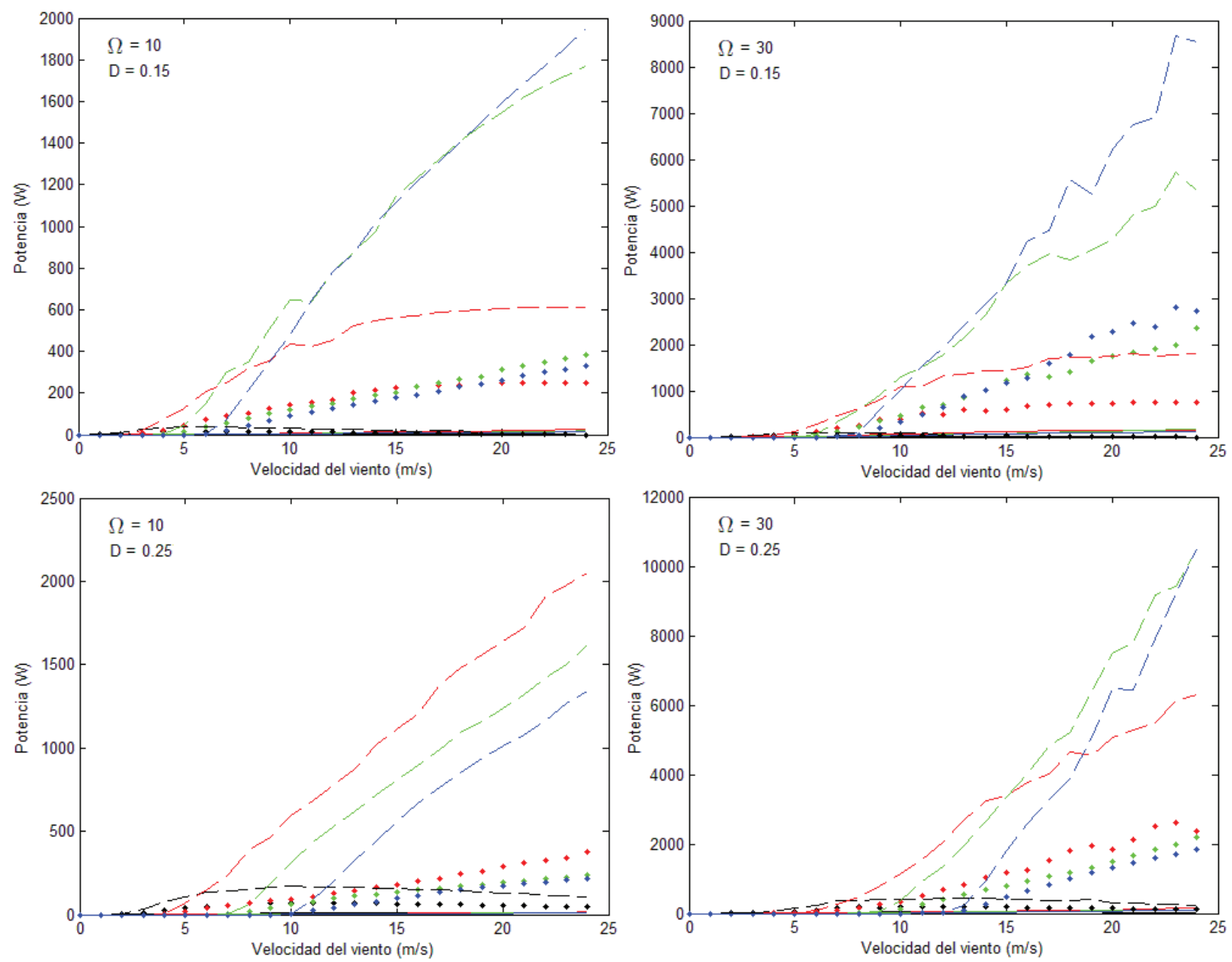

Figura 6. Curvas de potencia de la turbina Magnus de dos cilindros. En cada gráfico $\mathrm{L}=1 \mathrm{~m}$ (sólida), $2 \mathrm{~m}$ (puntos) y 3 m (discontinua); $\omega=1000$ RPM (negro), 4000 RPM (rojo), 8000 RPM (verde) y 12000 RPM (azul). D (m), $\Omega$ (RPM)

Se observa también en las figuras 5 y 6 que al aumentar la longitud de los cilindros se obtiene una mayor potencia de la turbina, del mismo modo que en las turbinas eólicas convencionales. Esto es fácilmente pronosticable, tomando en cuenta que la potencia disponible en el viento es proporcional al área barrida por los cilindros.

Cabe destacar que, a diferencia del aumento en la velocidad de rotación de los cilindros, en este caso no ocurre una modificación significativa en el valor de velocidad del viento donde da inicio la extracción de energía para cada combinación particular de las otras variables, por lo que hacer la turbina más grande no la hace ineficiente para bajas velocidades del viento, como sí ocurre cuando se aumenta $\omega$.

En cuanto al diámetro de los cilindros, se encuentra que al aumentar su valor también crece la potencia máxima entregada por la turbina, pero la extracción de energía se inicia a velocidades del viento mayores y el punto de máxima potencia también se corre a la derecha en el gráfico de potencia en función de la velocidad del viento.

Este es un comportamiento similar al obtenido cuando se modifica $\boldsymbol{\omega}$. La mejora en la captura de potencia se debe, por una parte, a que las fuerzas aerodinámicas sobre las aspas de turbinas eólicas son directamente proporcionales a la cuerda (ver ecuaciones 10 y 11) y en el 
caso de las turbinas Magnus, el diámetro corresponde con el concepto de cuerda de un aspa convencional.

Por otra parte, considerando la definición de $\bar{\omega}$, que en su numerador contiene tanto a $\omega$ como al radio del cilindro, resulta razonable que un aumento en el radio cause un efecto similar al de aumentar la velocidad angular del cilindro, pues en ambos casos se requiere un aumento en el denominador (que es la velocidad del viento) para mantener $\bar{\omega}=2$ constante y operar en el punto óptimo.

La variación en la velocidad angular de la turbina modifica la curva de potencia de modo que, conforme se aumenta $\Omega$ se requiere una mayor velocidad del viento para que la turbina extraiga energía, pero a la vez se obtiene como beneficio que la cota máxima de potencia se incremente.

Sin embargo, para valores de $\Omega$ similares a los que se emplean en turbinas eólicas convencionales, la potencia máxima se alcanza a valores de $U_{\infty}$ muy altos $(20-25 \mathrm{~m} / \mathrm{s})$, lo cual no es práctico para la mayoría de las aplicaciones. Por tanto, de manera preliminar se puede indicar que la turbina Magnus ofrece ventajas para $\Omega$ moderados o bajos, tal como proponen autores como Bychkov et. al (2007), quienes señalan que la turbina Magnus opera de forma adecuada a un tercio de la velocidad angular de las eólicas convencionales, resultado que se obtuvo también en este trabajo.

En cuanto a la cantidad de cilindros de la turbina y su efecto en el comportamiento, el análisis demuestra que la tendencia es la misma que al aumentar el diámetro del cilindro o la velocidad angular de la turbina. No obstante, cada cilindro representa más material, mayor complejidad de construcción y un nuevo consumo de energía para generar la rotación, por lo que en este parámetro se debe incluir una nueva variable, que es el gasto energético necesario para la operación de la turbina en función de la cantidad de cilindros.

\section{Conclusiones}

Se requiere una velocidad de rotación del orden de $10^{3}$ RPM de las aspas cilíndricas de una turbina tipo Magnus para lograr extraer potencia del viento, lo que coincide con lo obtenido en investigaciones anteriores.

La variable que gobierna el comportamiento de esta turbina es la velocidad relativa de rotación del cilindro $\bar{\omega}=\omega r \mathrm{r}_{\infty}$. En concordancia con otros autores, se encuentra que para un rendimiento óptimo, la turbina debe operar en valores cercanos a $\bar{\omega}=2$.

La turbina Magnus resulta eficiente con un bajo número de cilindros, dado que al aumentar la cantidad de aspas cilíndricas, disminuye el máximo coeficiente de potencia alcanzable.

La eficiencia de este tipo de turbinas es directamente proporcional al diámetro del cilindro para un radio de turbina fijo, lo que no había sido reportado anteriormente en la bibliografía.

Acorde con lo señalado por otros autores, la turbina Magnus opera de forma adecuada a velocidades angulares menores que las eólicas convencionales de eje horizontal.

Recomendaciones para trabajo futuro

- Valorar si la turbina Magnus ofrece ventajas de emplazamiento respecto de las eólicas convencionales, enfocándose en las características de la estela que genera la turbina.

- Realizar simulaciones del desempeño de este tipo de generador inmerso en otro fluido, como el agua, para conocer su potencial de aplicación hidrocinético.

- Construir prototipos y realizar mediciones experimentales, tanto en aire como en agua. 


\section{Agradecimientos}

Se agradece al Instituto Tecnológico de Costa Rica y al Ministerio de Ciencia, Tecnología y Telecomunicaciones de Costa Rica, por el financiamiento parcial para esta investigación. Al Arq. Diego Murillo Calderón, por su colaboración con los diagramas.

\section{Bibliografía}

Burton, T., Sharpe, D., Jenkins, N. \& Bossanyi, E. (2001). Wind energy handbook. John Wiley \& Sons.

Bychkov, N.M. (2008). Magnus wind turbine. 3. Calculated characteristics of the windwheel. Thermophysics and Aeromechanics, 15(2), 321-331.

Bychkov, N.M., Dovgal, A.V. \& Kozlov, V.V. (2007). Magnus wind turbines as an alternative to the blade ones. En Journal of Physics: Conference Series, 75, No. 1, p. 012004. IOP Publishing.

Ceyhan, O.Z.L.E.M. (2008). Aerodynamic design and optimization of horizontal axis wind turbines by using BEM theory and genetic algorithm. Doctoral dissertation, Master Thesis, Aerospace Engineering Department, METU, Ankara.

Fei, F., Mai, J.D. \& Li, W.J. (2012). A wind-flutter energy converter for powering wireless sensors. Sensors and Actuators A: Physical, 173(1), 163-171.

Goňo, R., Rusek, S. \& Hrabčík, M. (2009, May). Wind turbine cylinders with spiral fins. En 8th EEEIC International Conference on Environment and Electrical Engineering, Karpacz, Poland (pp. 10-13).

Islam, M., Ting, D.S.K. \& Fartaj, A. (2008). Aerodynamic models for Darrieus-type straight-bladed vertical axis wind turbines. Renewable and Sustainable Energy Reviews, 12(4), 1087-1109.

Ito, A., Kawashima, S., Nishizawa, Y., Ushiyama, I. \& Komatinovic., N. (2007). A study on savonius type magnus wind turbine. Europe premier wind energy event. Obtenido de http://www.ewea.org/ewec2007/allfiles2/202_ Ewec2007fullpaper.pdf

Luo, D., Huang, D. \& Wu, G. (2011). Analytical solution on Magnus wind turbine power performance based on the blade element momentum theory. Journal of Renewable and Sustainable Energy, 3(3), 033104.

Manwell, J.F., McGowan, J.G. \& Rogers, A.L. (2010). Wind energy explained: theory, design and application. John Wiley \& Sons.

Massaguer, A., Massaguer, E., Pujol, T., Comamala, M. \& Velayos, J. (2014). Blade shape influence on aerodynamic efficiency of a Magnus wind turbine using particle image velocimetry. Renewable Energy and Power Quality Journal, 12, 1-6.

Moriarty, P.J. \& Hansen, A.C. (2005). AeroDyn theory manual. Golden, Colorado, USA: National Renewable Energy Laboratory.

Perković, L., Silva, P., Ban, M., Kranjčević, N. \& Duić, N. (2013). Harvesting high altitude wind energy for power production: The concept based on Magnus' effect. Applied Energy, 101, 151-160.

Sedaghat, A. (2014). Magnus type wind turbines: Prospectus and challenges in design and modelling. Renewable Energy, 62, 619-628.

Sun, X., Zhuang, Y., Cao, Y., Huang, D. \& Wu, G. (2012). A three-dimensional numerical study of the Magnus wind turbine with different blade shapes. Journal of Renewable and Sustainable Energy, 4(6), 063139. 